DESY 01-026

ISSN 0418-9833

hep-ph/0103056

March 2001

\title{
Photoproduction of Jets on a Virtual Pion Target in Next-to-Leading Order QCD
}

\author{
M. Klasen and G. Kramer \\ II. Institut für Theoretische Physik, Universität Hamburg, \\ Luruper Chaussee 149, 22761 Hamburg, Germany
}

\begin{abstract}
Differential cross sections for inclusive dijet photoproduction on a virtual pion have been calculated in next-to-leading order QCD as a function of $E_{T}, \eta$, and $x_{\pi}$. The cross sections are compared with recent ZEUS data on photoproduction of dijets with a leading neutron in the final state.
\end{abstract}

\section{Introduction}

Recently the ZEUS collaboration at HERA presented differential cross section data for the neutron tagged process $e^{+}+p \rightarrow e^{+}+n+$ jet + jet $+\mathrm{X}$ [1]. The cross sections have been measured in the photoproduction region with photon virtuality $Q^{2}<4 \mathrm{GeV}^{2}$ for $\gamma p$ center-of-mass energy $W$ in the interval $134<W<269 \mathrm{GeV}$, for jets with transverse energy $E_{T}>6 \mathrm{GeV}$, neutron energy $E_{n}>400 \mathrm{GeV}$, and neutron production angle $\Theta_{n}<0.8$ mrad. The cross sections were compared to predictions with the one-pion exchange model and rather good agreement was found.

Due to the kinematic constraints on the neutron detection the squared momentum transfer $t$ between ingoing proton and outgoing neutron is very small [1]. In this case it is expected that the $p \rightarrow n$ transition amplitude, i.e. the amplitude for the production of neutrons, which carry most of the momentum of the proton, is dominated by the lightest particle in the $t$-channel, the pion. Hence the isolation of the one-pion exchange contribution provides the possibility to study the photon-pion interaction $\gamma+\pi \rightarrow X$ [2].

Selecting high $E_{T}$ jets in the final state $X$ allows us to apply the QCD improved parton model to describe the characteristic features of the jet production dynamics. This is completely analogous to the process $\gamma+p \rightarrow$ jet + jet $+\mathrm{X}$, which has been calculated in the 
past up to next-to-leading order (NLO) using different computational techniques [3, 4]. The only difference in $\gamma+\pi \rightarrow$ jet + jet $+\mathrm{X}$ is that the parton distribution functions (pdfs) of the proton are replaced by the pdfs of the pion. In addition, the pion flux, generated by the $p \rightarrow n$ transition, has to be supplied.

The pion flux can, in principle, be measured in charge-exchange processes in soft hadronic reactions, where an initial-state proton is transformed into a final-state neutron, $p \rightarrow n$, with small momentum transfer. A successful phenomenological description of the corresponding data has been obtained in the framework of reggeized isovector exchange, such as $\pi, \rho$, and $a_{2}$, with the pion being by far the lightest meson dominating the $p \rightarrow n$ transition, particularly at small values of the squared momentum transfer $t$ between proton and neutron [5].

The pdfs of the pion can be measured in deep-inelastic scattering with a forward going neutron detected in the final state: $e^{+}+p \rightarrow e^{+}+n+X$ [6]. The measurements of the structure functions so far are limited to rather small Bjorken- $x$ [7, 8]. The measured semi-inclusive cross sections for leading neutrons by the $\mathrm{H} 1$ collaboration [8] can be described entirely by $\pi$ exchange, and the Bjorken- $x$ and $Q^{2}$ dependence of these data are consistent with leading order (LO) pdfs of the pion. There exist several such pion pdfs in the literature [9, 10, 11]. They are constrained by dimuon and prompt-photon production data from fixed target experiments that are sensitive to the valence quark distribution in a Bjorken- $x$ range relevant for dijet photoproduction on pions.

In this work we present the results of a calculation of differential cross sections for the reaction $e^{+}+p \rightarrow e^{+}+n+$ jet + jet $+\mathrm{X}$ with the kinematical constraints as in the ZEUS analysis [1]. We have done these calculations in LO and in NLO. It is well known, however, that LO predictions are not reliable due to the possibly strong scale dependence of the results. Another problem of LO results for jet cross sections is the fact that in LO only two large $E_{T}$ jets with opposite transverse momenta can be produced. Therefore these cross sections are independent of the jet algorithm which is applied in order to define jets in the analysis of the experimental data. In NLO calculations there appears an explicit dependence on the jet algorithm due to the possible production of three partons (in addition to the remnant partons on the photon or pion side, respectively) in the final state, two of which must be combined in one jet in accordance with the experimental constraints for the construction of jets out of hadrons. Therefore our LO results are just for comparison and for demonstrating the reduced scale dependence of the NLO results. Our genuine predictions are the NLO cross sections which will be compared with the ZEUS data [1].

In the next section we shall describe the kinematical variables and define our input for the pion flux and the pion pdfs. Section 3 contains our results and the comparison with 
the experimental data. In the last section we give a short summary and draw some conclusions.

\section{Kinematical Variables and Further Input}

The event kinematics has been described in detail already in [1]. Here we repeat those variables which are needed in the calculation of the cross cross sections. The reaction

$$
e^{+}(k)+p(P) \rightarrow e^{+}\left(k^{\prime}\right)+n\left(P^{\prime}\right)+H,
$$

where $\mathrm{H}$ is the hadronic system containing the jets, is characterized by the four-momenta $k$ and $k^{\prime}$ of the initial and scattered positron and by $P$ and $P^{\prime}$, the four-momenta of the ingoing proton and outgoing neutron, respectively. The positron-photon vertex is described by the exchanged photon virtuality $Q^{2}$ and the positron's inelasticity $y$, i.e.

$$
\begin{aligned}
Q^{2} & =-q^{2}=-\left(k-k^{\prime}\right)^{2}, \\
y & =\frac{P q}{P k} .
\end{aligned}
$$

In the ZEUS experiment [1] $Q^{2} \leq 4 \mathrm{GeV}^{2}$ and $0.2<y<0.8$, corresponding to the $\gamma p$ center-of-momentum energy range $134<W<269 \mathrm{GeV}$. The protons at HERA have the energy $E_{p}=820 \mathrm{GeV}$ and collide with $E_{e}=27.5 \mathrm{GeV}$ positrons, corresponding to a center-of-momentum frame energy $\sqrt{S}=300 \mathrm{GeV}$. The two variables, which describe the proton-neutron vertex, are the fraction of the energy of the initial-state proton carried by the neutron $x_{L}$ and the square of the momentum transfer $t$ between the proton and the produced neutron:

$$
\begin{aligned}
x_{L} & =\frac{P^{\prime} k}{P k} \simeq \frac{E_{n}}{E_{p}}, \\
t & =q^{\prime 2}=\left(P-P^{\prime}\right)^{2} .
\end{aligned}
$$

The splitting function or pion flux for the transition $p \rightarrow n+\pi^{+}$is usually parameterized by different forms which can be summarized as

$$
f_{\pi / p}\left(x_{L}, t\right)=\frac{1}{4 \pi} \frac{g_{n \pi p}^{2}}{4 \pi} \frac{-t}{\left(m_{\pi}^{2}-t\right)^{2}}\left(1-x_{L}\right)^{1-2 \alpha_{\pi}(t)}\left[F\left(x_{L}, t\right)\right]^{2} .
$$

Here $g_{n \pi p}$ is the coupling constant of the $n \pi p$ vertex, $m_{\pi}$ is the pion mass, and $\alpha_{\pi}(t)=$ $\alpha^{\prime}\left(t-m_{\pi}^{2}\right)$ is the Regge trajectory of the pion. $F\left(x_{L}, t\right)$ is a form factor which describes the off-shell behavior of the virtual pion and/or possible final state rescattering effects of the neutron. Two choices for the form factor $F\left(x_{L}, t\right)$ used in the analysis of various charge-exchange scattering experiments are

$$
F\left(x_{L}, t\right)=\left\{\begin{array}{l}
\exp \left[b\left(t-m_{\pi}^{2}\right)\right] \\
\exp \left[R^{2}\left(t-m_{\pi}^{2}\right) /\left(1-x_{L}\right)\right]
\end{array}\right.
$$


where $b$ and $R$ are constants. The first of these choices, the exponential form, is usually taken with the Regge trajectory factor in (4) with $\alpha^{\prime}=1 \mathrm{GeV}^{-2}$. The second choice, the light-cone form factor, is usually associated with the flux without the Regge trajectory factor, i.e. $\alpha^{\prime}=0$ in (4). These choices are associated with the experimental finding that in soft hadronic reactions the shape of the $x_{L}$ distribution depends on $t$ [12]. The pion-nucleon coupling constant is well known from low energy $\pi N$ and $N N$ scattering data. We take $g_{n \pi p}^{2} / 4 \pi=2 \cdot 14.17$ as obtained in a recent analysis [13].

In the scattering process of $2 \rightarrow 2$ massless partons, the fractions of the four-momenta $q=k-k^{\prime}$ and $q^{\prime}=P-P^{\prime}$ participating in the hard scattering by the initial state parton are given by

$$
\begin{aligned}
& x_{\gamma}=\frac{\sum_{j} E_{T}^{j} e^{-\eta_{j}}}{2 y E_{e}}, \\
& x_{\pi}=\frac{\sum_{j} E_{T}^{j} e^{\eta_{j}}}{2 E_{p}\left(1-x_{L}\right)},
\end{aligned}
$$

where the sums in (6) and (7) run over the variables of the two jets in the final state. Here we assumed that $q^{2}=q^{\prime 2}=0$. The energy fraction contributing by the exchanged virtual photon to the production of the dijets is $x_{\gamma}$ whereas the corresponding contribution of the virtual pion (or possibly of a reggeized $\rho$ or $a_{2}$ ) is $x_{\pi}$. In (6) $E_{\gamma}=y E_{e}$ is the energy of the ingoing virtual photon. In NLO also three jets can be produced in the final state. Then (6) and (7) are no longer valid. In order to estimate the energy fractions $x_{\gamma}$ and $x_{\pi}$ for this case, too, one uses (6) and (7) in the form that the sums in (6) and (7) run over the two jets of largest $E_{T}$ in an event.

As is well known two mechanisms contribute to the photoproduction of jets, the direct and the resolved process. The observable $x_{\gamma}$ is sensitive to the amount of direct and resolved processes. The LO direct process, where the photon couples directly to the quarks, contributes at $x_{\gamma}=1$, the resolved and the NLO direct processes contribute in the region $x_{\gamma}<1$. It must be emphasized, however, that the distinction of direct and resolved processes looses its meaning in NLO, so that the characterization of the contributions to the various $x_{\gamma}$ regions by the direct and resolved processes is not unique: It becomes scale dependent. The variable $x_{\pi}$ can be used to analyze which region of the pion pdfs and which partonic contributions are most important for dijet production with leading neutrons.

As for the jet definition and the combination of two partons into one jet in the NLO contributions of the direct and the resolved parts, we use the $k_{T}$ cluster algorithm in the form as introduced in [14] and as used in the jet analysis of the experimental data [1]. The photon flux is calculated with the usual Weizsäcker-Williams approximation, including the non-logarithmic correction as calculated in [15]. For the resolved cross sections we 
need the pdfs of the photon in LO and NLO. A popular parameterization is GRV [16]. We use the parameterization GS96 [17], since it leads to a slightly better description of the experimental data. For the comparison with data of inclusive jet production without the leading neutron, which has been simultaneously analyzed in [1] under similar kinematical conditions, we need also the pdfs of the proton, for which we employ the recent CTEQ5M [18 parameterization. The $\Lambda$ parameter which we need in the NLO $\alpha_{s}$ formula is taken from the proton pdf fit. It is $\Lambda^{(4)}=326 \mathrm{MeV}$. For the pion pdfs we employ two alternatives, SMRS3 9] and GRS [11]. Unfortunately, the $\Lambda$ values used for the evolution of these pdfs are somewhat smaller, namely $\Lambda^{(4)}=190$ and $200 \mathrm{MeV}$. For LO predictions, we use LO matrix elements with the one-loop formula for $\alpha_{s}$ and the same values of $\Lambda$. Since only GRS have constructed LO and NLO pion pdfs, we have to use the NLO SMRS3 set also with our LO predictions. The GRS pdfs of the pion are constructed for three flavors only. Since we include four flavors throughout, we also need the pion pdf for charm. This is taken from the earlier work of GRV [10].

\section{$3 \quad$ Results and Comparison with ZEUS Data}

The calculation of the cross sections is based on the formalism fully described in our previous work [3]. To cancel infrared and collinear singularities in the NLO contributions of the final state and to define the collinear initial state singularities to be removed through the renormalization of the pdfs of the photon or the pion, the phase-space slicing method was applied. Since the neutron kinematics could not be fixed in detail in the experiment we had to integrate over a finite region in $x_{L}$ and $t$. We did this in accordance with the specifications of the ZEUS experimental analysis. Except for the outgoing positron and the leading neutron the final state consists of two or three jets. The two-jet sample contains the bare parton jets from the LO and virtual NLO contributions and the two jets originating from the recombination of two partons in the three-parton terms. The three-jet sample is just given by the uncombined three-parton final states.

First we calculated the differential cross section $d^{2} \sigma / d E_{T} d \eta$, where $E_{T}$ and $\eta$ are the transverse energy and the rapidity of one of the jets in the two- or three-jet sample, respectively. As in the ZEUS analysis we restricted the selection in the three-jet sample. We included from this sample only the two jets with the largest $E_{T}$. In our earlier work [3] this cross section was denoted the inclusive one-jet cross section. There, however, we included all three jets from the three-jet contributions. In [1] the cross section defined above is denoted the dijet cross section. This should be distinguished from the inclusive two-jet cross section defined in [3], which depends on $E_{T}$, the transverse energy of one of the two jets with the highest $E_{T}$, and the rapidities $\eta_{1}$ and $\eta_{2}$ of these high $E_{T}$ jets. The $d^{2} \sigma / d E_{T} d \eta$ is integrated over $-2<\eta<2$ as in [1]. The result for the full jet sample (without detection of the leading neutron) as a function of $E_{T}$ for $E_{T}>6 \mathrm{GeV}$ turn out 
to be in reasonable agreement with the experimental data from [1] (not shown). The point with the largest cross section at $E_{T}=7 \mathrm{GeV}$ agrees perfectly with the measured value. The points at medium $E_{T}$ lie somewhat higher than the theoretical curve. The equivalent cross section $d^{2} \sigma / d E_{T} d \eta$, also integrated over $|\eta|<2$, for the case with the leading neutron is shown as a function of $E_{T}$ for $6.5<E_{T}<29.5 \mathrm{GeV}$ in Fig. 1a. In this figure three curves are shown: one from LO with pion pdfs SMRS3 [9] and photon pdfs GS96 [17], the latter in LO, and two other curves for NLO with pion pdfs SMRS3 [9] and GRS [11] and charm content from GRV [10] for comparison. We see that all three predictions lie very near to each other in the logarithmic plot. The LO and NLO curves for SMRS3/GS96 differ only very little in this plot, showing that the NLO corrections are small for the scale choice $\mu=\max \left(E_{T, 1}, E_{T, 2}\right)$ as used in Fig. 1a (and in all the following figures) for the LO and NLO result. The GRS choice for the pion pdfs leads to a somewhat larger cross section at small $E_{T}$ 's and smaller cross section at the larger $E_{T}$ 's as compared to the SMRS3 choice. We have calculated that the gluon in the pion gives the dominant contribution to the cross section for $E_{T}<22 \mathrm{GeV}$. Resolved photons are more important than direct photons for $E_{T}<9 \mathrm{GeV}$. The experimental data [1] with statistical and systematic errors shown separately come with a light shaded band which indicates the additional systematic experimental uncertainty due to the calorimeter energy scale [1]. The medium shaded band gives the uncertainty of the LO result when the scale of $\alpha_{s}$ and of the factorization is varied in the interval between $E_{T} / 2$ and $2 \cdot E_{T}$. The scale uncertainty of the NLO prediction (dark shaded band) coincides with the line thickness of the NLO curve. Taking into account the fairly large experimental errors including the calorimeter energy scale uncertainty the agreement of the ZEUS data with the theoretical predictions concerning shape and absolute normalization is reasonably good. It must be emphasized, however, that the absolute normalization depends on the pion form factor (5). In Fig. 1a we assumed the light-cone form factor with $R=0.5 \mathrm{GeV}^{-1}$. Recent determinations of $R^{2}$ from a comparison to the rather accurate neutron production data from Flauger and Mönning [12] yield $R^{2}=0.2 \mathrm{GeV}^{-2}$ [5] and $R^{2}=0.4 \mathrm{GeV}^{-2}$ [19] in reasonable agreement with our value for $R^{2}$. A change of $R$ from $R=0.5$ to $R=0.6 \mathrm{GeV}^{-1}$, i.e. an increase by $20 \%$, leads to a $15 \%$ smaller cross section.

Since we have calculated also $d^{2} \sigma / d E_{T} d \eta$ with the same kinematical constraints for $e+p \rightarrow e^{\prime}+2$ jets $+\mathrm{X}$ we can take the ratio of the two cross sections $d \sigma / d E_{T}$ for $e+p \rightarrow e^{\prime}+n+2$ jets $+\mathrm{X}$ and $e+p \rightarrow e^{\prime}+2$ jets $+\mathrm{X}$. This is shown in Fig. 1b and compared to the ZEUS data for this ratio. The agreement with the data is perfect. For low $E_{T}$, where the experimental errors are smallest, the SMRS3 result agrees better than the ratio obtained with the GRS pion pdfs, otherwise the results are the same. In Fig. 1b the experimental uncertainty from the calorimeter energy measurements, the systematic error and the scale uncertainty in the theoretical curve are not included since they are supposed to cancel in the ratio [1]. Thus, this ratio seems to be a good example to get information on the pion pdfs and/or the pion form factor. 

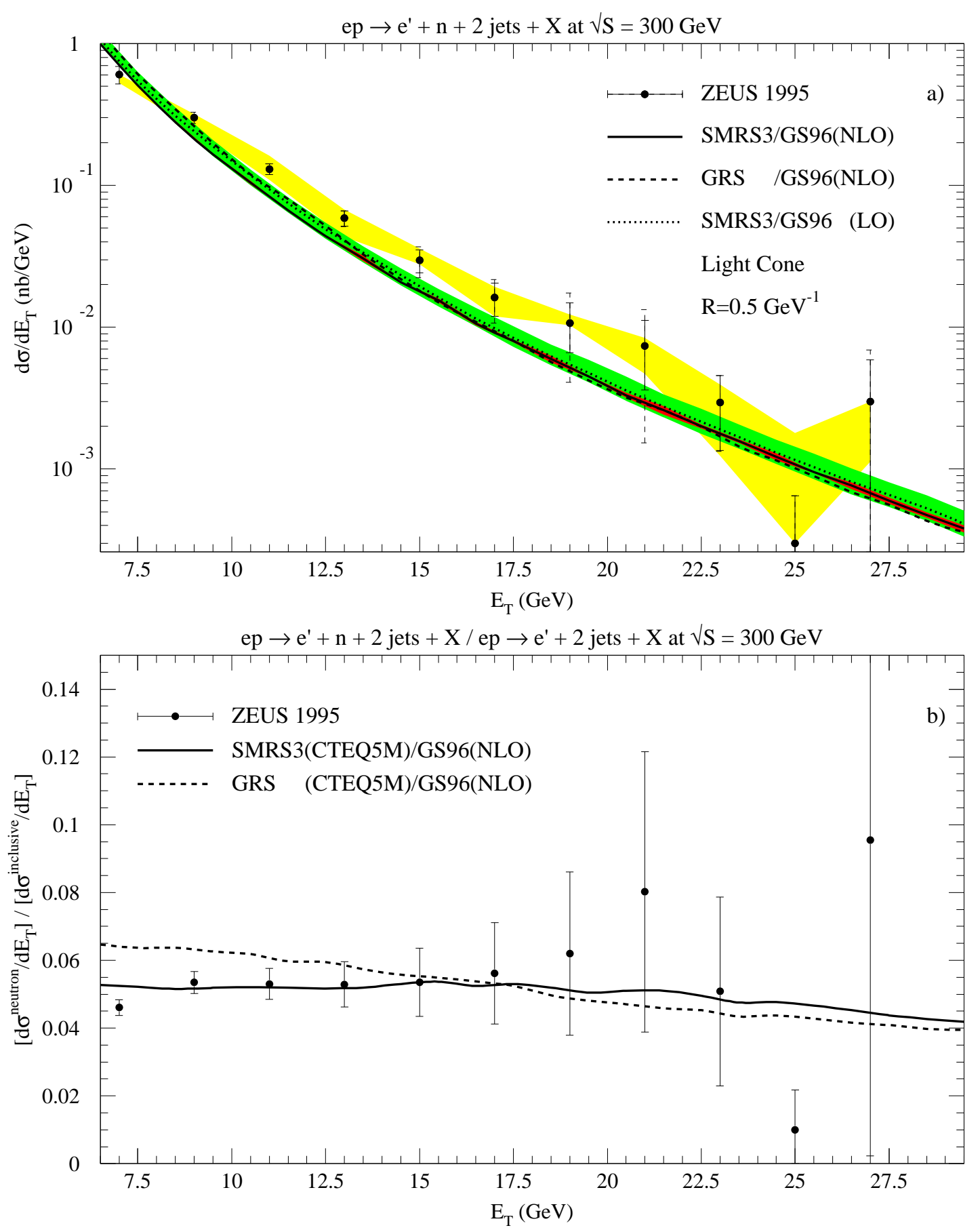

Figure 1: Dependence of the dijet photoproduction cross section with a leading neutron on the transverse energy of one of the two jets (a). Two NLO predictions with different pion structure functions and one LO prediction are shown together with experimental data from ZEUS with statistical (inner) and systematic (outer) experimental error bars. In addition, error bands from the theoretical scale uncertainty (medium: LO, dark: NLO) and the experimental energy scale uncertainty (light) are shown. The NLO error band coincides with the thickness of the full line. In b) we present the ratio of the leading neutron over the inclusive cross section versus the transverse energy. NLO calculations with two different pion structure functions are shown together with the experimental ZEUS data. In this case, only statistical error bars are given. 
The next calculated differential cross section is $d \sigma / d \eta$, i.e. $d^{2} \sigma / d E_{T} d \eta$ integrated over $E_{T}$ with $E_{T, \min }=6 \mathrm{GeV}$. The result is shown in Fig. 2a, where $d \sigma / d \eta$ is plotted as a function of $\eta$ for $\eta$ between -2 and 2, together with the data points from [1]. There are three theoretical curves for the three cases as in Fig. 1a. Here one can see the difference between SMRS3/GS96 in LO and NLO. The difference is very small, the LO cross section is approximately $5 \%$ larger than the NLO cross section for the same scale $\mu=\max \left(E_{T, 1}, E_{T, 2}\right)$. Concerning the dependence on the pion pdfs the choice SMRS3 agrees best with the data. The result with the GRS pdfs is larger, in particular for negative $\eta$. In [9] two other pion pdfs have been published, SMRS1 and SMRS2. The cross sections for these two pdfs lie between the SMRS3 and the GRS curves and are therefore not shown explicitly. The gluons in the pion contribute about twice as much as quarks to the rapidity distribution over the entire $\eta$-range. Direct (resolved) photons dominate in the region $\eta<0(\eta>0)$. All three curves do not agree well with the data points for $\eta<-0.5$. For jet production without leading neutron this region is known to be influenced by hadronization corrections [20]. We expect such corrections also for the cross section in Fig. 2a, although somewhat less in the case with leading neutron due to the reduced total energy producing the hard jets. The theoretical result with such hadronic corrections included (taken from the case without leading neutron 200) is shown in Fig. 2a as the lower full curve. It leads to a better agreement in the negative $\eta$ region, but it produces also a somewhat lower cross section at positive $\eta$ 's, reducing the good agreement wit the data in this region. In Fig. 2a we show again three shaded bands: (i) the light shaded band shows the additional systematic uncertainty from the calorimeter energy scale, (ii) the medium shaded band gives the variation of the LO result (dotted curve) with the scale $\mu$, which was varied as usual in the region $E_{T} / 2<\mu<2 \cdot E_{T}$, (iii) the dark shaded band shows the variation of the NLO cross section with changing the scale in the same interval. Here, we see quite clearly that the NLO result has a very much reduced scale dependence compared to the LO result. Similarly to Fig. 1b we have plotted in Fig. 2b the ratio of the cross sections for SMRS3/GS96 and GRS/GS96 and the cross section $d \sigma / d \eta$ for dijet production without leading neutron as a function of $\eta$ and compared them with the same experimental ratio from ZEUS. Within errors the SMRS3 and the GRS results are consistent with the data, the SMRS3 curve agrees slightly better.

The last differential cross section which we investigated is $d \sigma / d \log _{10}\left(x_{\pi}\right)$ as a function of $\log _{10}\left(x_{\pi}\right)$. In order to determine $x_{\pi}$, which is a measure of the exchanged pion's momentum fraction participating in the production of jets as defined in (7), we need the transverse momenta and rapidities of the two most energetic jets in the event. The experimental cross section $d \sigma / d \log _{10}\left(x_{\pi}\right)$, however, has the constraint that for the two jets $E_{T, 1} \geq 6 \mathrm{GeV}$ and $E_{T, 2} \geq 6 \mathrm{GeV}$. Cross sections for $E_{T, 1}=E_{T, 2}$ are problematic theoretically, since they become infrared sensitive in NLO, i.e. depend on the slicing cut used to cancel infrared and collinear singularities. In order to avoid this sensitivity and also possible hadronic and intrinsic $k_{T}$ effects not considered in our NLO framework we need constraints on $E_{T, 1}, E_{T, 2}$ or $E_{T, 3}$ which smear out the problematic region $E_{T, 1}=E_{T, 2}$. 

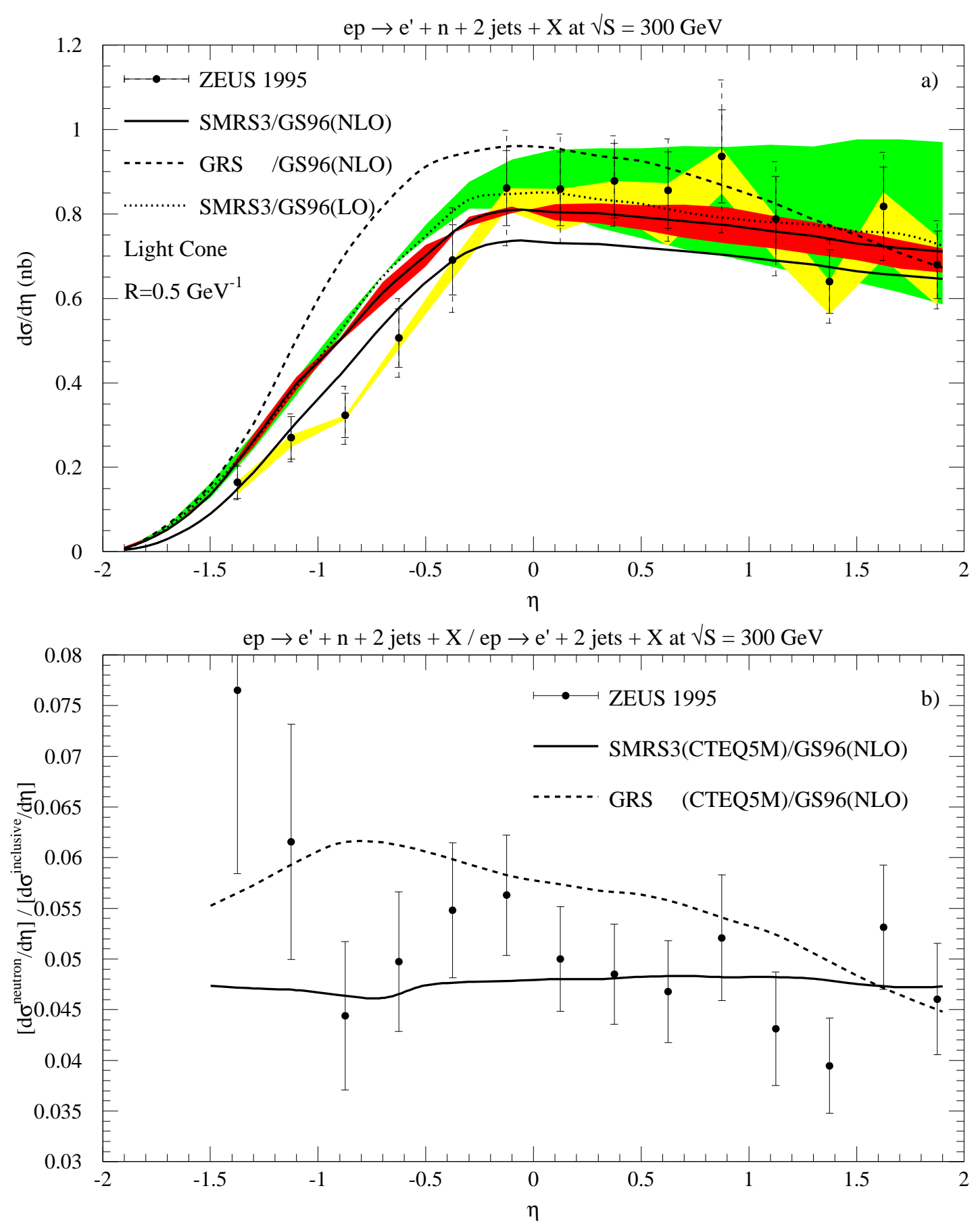

Figure 2: Dependence of the dijet photoproduction cross section with a leading neutron on the rapidity of one of the two jets (a). In b) we show the ratio of the leading neutron over the inclusive cross section versus the rapidity. Details are as in Fig. 1. The lower full curve in a) demonstrates the influence of hadronization corrections. 


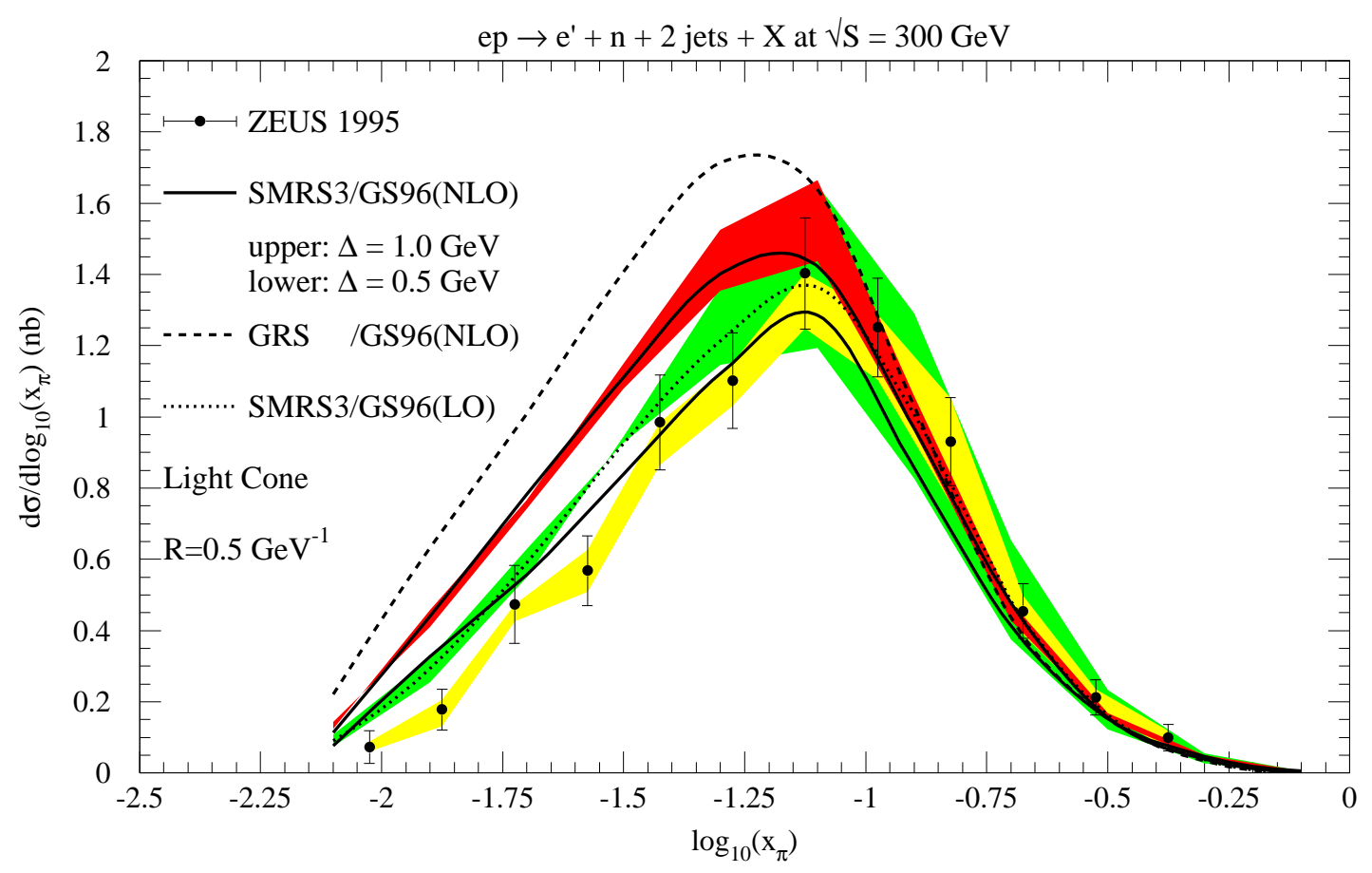

Figure 3: Dependence of the dijet photoproduction cross section with a leading neutron on the logarithm of the observed momentum fraction of the partons in the pion $\log _{10}\left(x_{\pi}\right)$. Details are as in Figs. 1a and 2a. In addition, we show the effect of lowering the transverse energy difference $\Delta$ from $1 \mathrm{GeV}$ to $0.5 \mathrm{GeV}$.

This problem is well known and was encountered some time ago also in connection with the calculation of the inclusive dijet cross section in $\gamma p$ collisions 21. One remedy to remove the infrared sensitivity is the requirement of slightly different lower limits on $E_{T, 1}$ and $E_{T, 2}$. Therefore, we have calculated $d \sigma / d \log _{10}\left(x_{\pi}\right)$ with the constraint $E_{T, 1} \geq 6 \mathrm{GeV}$, $E_{T, 2} \geq 5 \mathrm{GeV}$ if $E_{T, 1}>E_{T, 2}$ or $E_{T, 1}$ and $E_{T, 2}$ interchanged if $E_{T, 2}>E_{T, 1}$. The cross sections calculated with this constraint are plotted in Fig. 3, again for the three cases as in Fig. 1a. These cross sections are compared with the corresponding ZEUS data. The NLO prediction with the pion pdfs of SMRS3 agrees better with the data than the one with GRS pion pdfs. Except for large $x_{\pi}>0.35$ the gluons in the pion dominate over quarks, and the resolved photon contribution is larger than the direct one for $x_{\pi}>0.04$. The agreement with the data is good for $x_{\pi}>0.06$, but less good for the smaller $x_{\pi}$. This $x_{\pi}$ region is related to the negative $\eta$ region in the $\eta$ distribution in Fig. 2a, where one has hadronization corrections (see (7) for the relation between $x_{\pi}$ and the $\eta_{j}$ 's). We note that the $\mathrm{K}$ factor between the NLO and LO result is larger than in $d \sigma / d \eta$ and is larger than one now in particular for small $x_{\pi}$. This is connected with the specific constraint on $E_{T, 1}$ 
and $E_{T, 2}$, which was not necessary for the results in Figs. 1 and 2. This constraint does not exactly correspond to the one applied to the measured $d \sigma / d \log _{10}\left(x_{\pi}\right)$. On the other hand the low $E_{T}$ calorimeter jets used in the experimental analysis have an uncertainty of $5 \%$ which introduces a corresponding uncertainty on the constraint $E_{T, 1}, E_{T, 2} \geq 6 \mathrm{GeV}$. In order to see the influence of the $E_{T, 1}, E_{T, 2}$ cut we reduced the difference $\Delta$ between $E_{T, 1}$ and $E_{T, 2}$ from $1 \mathrm{GeV}$ to $0.5 \mathrm{GeV}$. The result is shown in Fig. 3 as the lower full curve. The reduction of the difference leads to a lower cross section as we expect, so that the low $x_{\pi}$ experimental points are described very well now but not the higher $x_{\pi}$ region. Since this region is much less affected by hadronization corrections we prefer the original prediction (upper full curve in Fig. 3). The absolute normalization of the cross section $d \sigma / d \log _{10}\left(x_{\pi}\right)$ depends also on the pion form factor. The curves in Fig. 3 are calculated with the light-cone form factor with $R=0.5 \mathrm{GeV}^{-1} . R=0.6 \mathrm{GeV}^{-1}$ instead gives a $15 \%$ lower cross section and better agreement for $x_{\pi}<0.06$ but worse agreement above this value. Again taking into account possible hadronization corrections we consider the value $R=0.5 \mathrm{GeV}^{-1}$ as more realistic. The result with $R=0.6 \mathrm{GeV}^{-1}$ is almost indistinguishable from the result with the exponential form factor with $b=0$.

In [1] several uncorrected distributions are presented, in particular event rates as a function of $x_{\gamma}$ in order to say something about the amount of resolved and direct photoproduction. From these rates, the ratio of resolved (corresponds to $x_{\gamma}<0.75$ ) to the direct cross section (corresponds to $x_{\gamma}>0.75$ ) has been deduced. We checked that the theoretical prediction for this ratio is in reasonable agreement with the data. It decreases with increasing $E_{T}$, as one expects it.

In this work we considered only the pion exchange in the $t$-channel. Other isovector exchanges, such as $\rho$ and $a_{2}$, are expected to be small. Recent studies of leading neutron production in $e p$ collisions show that the rate can be described entirely by $\pi^{+}$exchange [8]. The additional exchanges, which increase the rate of neutron production, are offset by absorptive rescattering of the neutron, which decreases the rate by approximately

the same amount [5]. Therefore both effects are neglected in the present analysis. If the experimental data were more specific concerning the dependence on $t$ and $x_{L}$, the dominance of the $\pi^{+}$exchange could be demonstrated.

\section{Summary and Concluding Remarks}

We have presented a next-to-leading order calculation of dijet production in collisions of almost real photons and pions, where the photons are generated through bremsstrahlung from an initial electron or positron beam and the pion is assumed to give the dominant contribution to the forward proton to neutron transition amplitude. Our results have been compared with recent data from ZEUS for distributions in the transverse energy $E_{T}$ and rapidity $\eta$ of one of the two jets and for the observed momentum fraction of the 
partons in the pion $x_{\pi}$. We found that the NLO corrections to the LO result were generally small, also in the case of inclusive photon-proton scattering with similar kinematic constraints, but lead to a largely reduced dependence on the renormalization and factorization scales. For the NLO distribution in $\log _{10}\left(x_{\pi}\right)$, we had to relax the experimental condition of $E_{T, 1}, E_{T, 2}>6 \mathrm{GeV}$ to allow for a small difference $\Delta=0.5 \ldots 1 \mathrm{GeV}$ of the two minimal transverse energies, corresponding to the non-zero experimental uncertainty in the jet energy scale.

The overall normalization of all three distributions is sensitive to the form factor of the $p \rightarrow n \pi^{+}$transition. We have used a light-cone form with a value for the parameter $R$ which agrees well with recent determinations from neutron production data. Once the normalization is fixed, the shapes of the distributions, particularly the one in $\log _{10}\left(x_{\pi}\right)$, are sensitive to the parton distributions in the pion. The ZEUS data for the distribution in $\log _{10}\left(x_{\pi}\right)$ as well as in $E_{T}$ and $\eta$ are best described by the SMRS3 pion parameterization, whereas the GRS parameterization with GRV charm distribution gives larger predictions, especially at small $x_{\pi}$. We emphasize that for small $E_{T}$ one is particularly sensitive to the gluon content of the pion. Furthermore, the GS96 photon densities agree better with the data than GRV. Hadronization corrections were found to reduce the perturbative prediction considerably in the region of small $\eta$, which corresponds to small $x_{\pi}$.

\section{Acknowledgments}

We thank M. Khakzad, G. Levman and J. Whitmore for making the ZEUS data available to us and M. Wing for calculating the hadronization corrections with the HERWIG Monte Carlo generator. Financial support by the Deutsche Forschungsgemeinschaft through Grant No. KL 1266/1-1, by the Bundesministerium für Bildung und Forschung through Grant No. 05 HT9GUA 3, and by the European Commission through the Research Training Network Quantum Chromodynamics and the Deep Structure of Elementary Particles under Contract No. ERBFMRX-CT98-0194 is gratefully acknowledged.

\section{References}

[1] J. Breitweg et al. [ZEUS Collaboration], DESY-00-142, October 2000, hepex/0010019.

[2] G. Kramer, Proc. of the Seminar on $e-p$ and $e-e$ Storage Rings, ed. by J.K. Bienlein, I. Dammann and H. Wiedemann, DESY-73-66, December 1973.

[3] M. Klasen and G. Kramer, Z. Phys. C 76 (1997) 67;

M. Klasen, T. Kleinwort and G. Kramer, Eur. Phys. J. direct C 1 (1998) 1. 
[4] B. W. Harris and J. F. Owens, Phys. Rev. D 56 (1997) 4007;

S. Frixione and G. Ridolfi, Nucl. Phys. B 507 (1997) 315;

P. Aurenche, L. Bourhis, M. Fontannaz and J. P. Guillet, Eur. Phys. J. C 17 (2000) 413.

[5] U. D'Alesio and H. J. Pirner, Eur. Phys. J. A 7 (2000) 109 and the earlier literature given there.

[6] J. D. Sullivan, Phys. Rev. D 5 (1972) 1732;

N. S. Craigie and G. Schierholz, Nucl. Phys. B 100 (1975) 125.

[7] M. Derrick et al. [ZEUS Collaboration], Phys. Lett. B 384 (1996) 388.

[8] C. Adloff et al. [H1 Collaboration], Eur. Phys. J. C 6 (1999) 587.

[9] P. J. Sutton, A. D. Martin, R. G. Roberts and W. J. Stirling, Phys. Rev. D 45 (1992) 2349.

[10] J. F. Owens, Phys. Rev. D 30 (1984) 943;

P. Aurenche, R. Baier, M. Fontannaz, M. N. Kienzle-Focacci and M. Werlen, Phys. Lett. B 233 (1989) 517;

M. Glück, E. Reya and A. Vogt, Z. Phys. C 53 (1992) 651.

[11] M. Glück, E. Reya and I. Schienbein, Eur. Phys. J. C 10 (1999) 313.

[12] J. Engler et al., Nucl. Phys. B 84 (1975) 70;

W. Flauger and F. Mönnig, Nucl. Phys. B 109 (1976) 347;

B. Robinson et al., Phys. Rev. Lett. 34 (1975) 1475;

G. Levman and K. Furutani, DESY-95-142, July 1995, and further references given there.

[13] T. E. Ericson, B. Loiseau and A. W. Thomas, hep-ph/0009312.

[14] S. Catani, Y. L. Dokshitzer, M. H. Seymour and B. R. Webber, Nucl. Phys. B 406 (1993) 187 ;

S. D. Ellis and D. E. Soper, Phys. Rev. D 48 (1993) 3160.

[15] S. Frixione, M. L. Mangano, P. Nason and G. Ridolfi, Phys. Lett. B 319 (1993) 339.

[16] M. Glück, E. Reya and A. Vogt, Phys. Rev. D 46 (1992) 1973.

[17] L. E. Gordon and J. K. Storrow, Nucl. Phys. B 489 (1997) 405.

[18] H. L. Lai et al. [CTEQ Collaboration], Eur. Phys. J. C 12 (2000) 375.

[19] H. Holtmann, A. Szczurek and J. Speth, Nucl. Phys. A 596 (1996) 631. 
[20] B. W. Harris, M. Klasen and J. Vossebeld, Proc. of the Workshop on Monte Carlo Generators at HERA Physics, Hamburg, Germany, 1998, hep-ph/9905348; M. Wing, priv. communication.

[21] M. Klasen and G. Kramer, Phys. Lett. B 366 (1996) 385. 